\title{
Dual-Labeling Strategies for Nuclear and Fluorescence Molecular Imaging: A Review and Analysis
}

\author{
Ali Azhdarinia, Pradip Ghosh, Sukhen Ghosh, Nathaniel Wilganowski, \\ Eva M. Sevick-Muraca
}

Center for Molecular Imaging, The Brown Foundation Institute of Molecular Medicine, The University of Texas Health Science Center, Houston, TX 77030, USA

\begin{abstract}
Molecular imaging is used for the detection of biochemical processes through the development of target-specific contrast agents. Separately, modalities such as nuclear and near-infrared fluorescence (NIRF) imaging have been shown to non-invasively monitor disease. More recently, merging of these modalities has shown promise owing to their comparable detection sensitivity and benefited from the development of dual-labeled imaging agents. Dual-labeled agents hold promise for whole-body and intraoperative imaging and could bridge the gap between surgical planning and image-guided resection with a single, molecularly targeted agent. In this review, we summarized the literature for dual-labeled antibodies and peptides that have been developed and have highlighted key considerations for incorporating NIRF dyes into nuclear labeling strategies. We also summarized our findings on several commercially available NIRF dyes and offer perspectives for developing a toolkit to select the optimal NIRF dye and radiometal combination for multimodality imaging.
\end{abstract}

Key words: Dual-labeling, Multimodality, Radiochemistry, Positron emission tomography, Near-infrared fluorescence, Single-photon emission computed tomography

\section{Introduction}

$\mathrm{T}^{\mathrm{t}}$ he advent of molecular imaging in clinical practice originates from radiopharmacology and is enabled by the exquisite sensitivity of devices and tracers that remain the "gold-standard" for molecular imaging approaches. Recently, advances in fluorescence imaging instrumentation and probe development promise new opportunities for its clinical implementation. As evidence of its emerging role in clinical studies, a recent literature search on PubMed shows that the number of papers describing the clinical use of nearinfrared fluorescence (NIRF) imaging has markedly increased in the past 5 years (Fig. 1). To date, all clinical NIRF imaging studies have been restricted to indocyanine

Correspondence to: Ali Azhdarinia; e-mail: ali.azhdarinia@uth.tmc.edu green (ICG) [1], the only dye which is approved for diagnostic use based upon intravenous administration for visualization of its dark green color. Despite its limitations as a fluorescent agent, which include (1) poor fluorescence properties arising from low quantum yield (QY), (2) small Stokes shift, (3) insufficient chemical and photostability in buffer or physiologic conditions, (4) the propensity to form aggregates in aqueous media, and (5) lack of a functional group for conjugation to targeting moieties, ICG has been used intraoperatively to assess blood vessel patency, tumordraining lymphatics and lymph node (LN) basins, and tumor margins (for review see [2-5]). ICG has also been used to non-invasively detect blood and lymphatic vasculatures, as well as LNs following microdose (i.e., 1/100 of the pharmacologic dose, $\leq 100 \mu \mathrm{g}$ of labeled protein, or $\leq 30 \mathrm{nmol}$ of labeled biologic agent) administration in humans [6]. Given the sensitivity demonstrated in these initial 


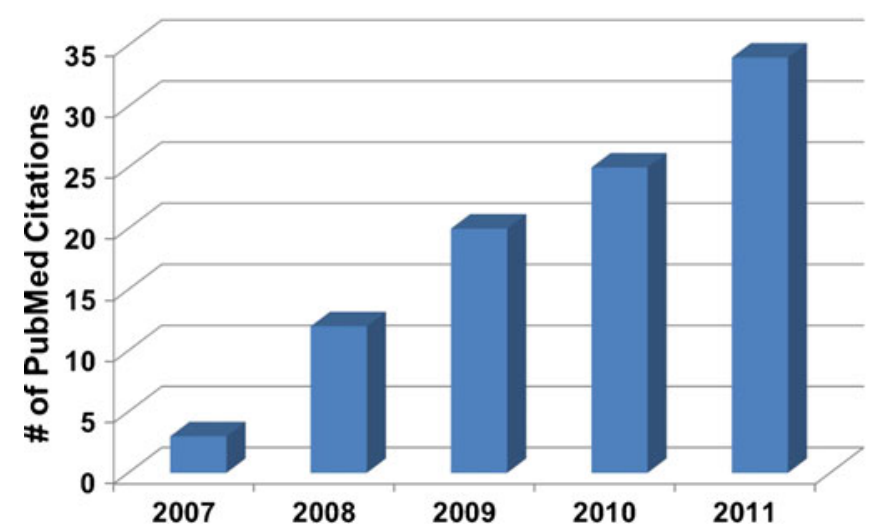

Fig. 1. Number of PubMed publications per year describing clinical NIRF studies (as of July 2011).

investigational human studies with ICG, new NIRF dyes with functional groups for bioconjugation now promise unique clinical opportunities for fluorescence-guided surgery with targeted molecular imaging. While nuclear imaging probes enable molecularly guided resection of tissues using audible detection, fluorescence arising from a localized targeting agent may enable real-time visual detection and permit image-guided resection of diseased tissues within the surgical field of view.

The success of NIRF molecular imaging critically depends upon demonstrating that its sensitivity for intraoperative and non-invasive molecular imaging rivals that offered by nuclear modalities. To provide a baseline by which NIRF imaging can be compared to nuclear imaging modalities, it is instructive to compare the physics of both modalities (Fig. 2). While nuclear imaging depends upon detecting the emission of highly penetrating gamma photons $(25-511 \mathrm{keV})$ produced in the course of radioactive decay, NIRF depends upon the selective and repeated activation of a fluorescent dye by tissue-penetrating excitation light and the collection of the low-energy emission photons. The radiative decay of a radiotracer occurs only once. In contrast, fluorescent dye molecules can experience repeated activation and radiative decay. Given a typical fluorescent lifetime, or the mean time between activation and radiative relaxation of $1 \mathrm{~ns}$, and given that $10 \%$ of all relaxations typically result in emitted radiation, the maximum photon count rate that a single fluorescent dye molecule can theoretically achieve is $100,000,000$ fluorescent photons/s [7]. Unfortunately, the emitted low-energy $(\sim 1.5 \mathrm{eV})$ photons are multiply scattered during tissue transit, creating a tradeoff between the amount of fluorescent photons generated in deeper tissues and the amount detected at tissue surfaces. Because attenuation of light is minimal at tissue surfaces and subsurfaces within the surgical field, fluorescent dyes may provide greater sensitivity than the clinical standard of radiotracers for intraoperative guidance; hence "first-in-humans" NIRF molecular targeting agents are more likely to have their initial clinical application occur in the surgical suite.

Unlike nuclear imaging where emitted photons can be readily detected regardless of depth, the attenuation of NIR fluorescent light emanating from deep tissues limits the sensitivity of fluorescence imaging and tomography as compared to its more established nuclear counterpart. Nonetheless, targeted NIRF conjugates have shown high sensitivity for in vivo preclinical molecular imaging, permitting agent administration at levels comparable to that of radiotracers (i.e., high $\mathrm{fM}-\mathrm{pM}$ of administered agent) [8], and enabling dual-labeling strategies. Dual-labeled imaging agents capable of (1) whole-body or regional nuclear imaging for surgical planning, and (2) NIRF imaging for molecularly guided surgery could improve patient outcomes by combining capabilities for diagnostic radiology with image-guided surgery in a single-imaging agent. Moreover, the addition of a NIRF dye onto a radiotracer enables the definitive correlation between the imaging used for surgical planning and the imaging used to direct surgery. Besides their clinical utility, dual-labeled imaging agents provide a method to quantitatively and definitively validate preclinical NIRF tomography using standard positron emission tomography (PET) or single-photon emission computed tomography (SPECT) values of percent injected dose per gram of tissue as "ground truth" in hybrid imaging. While the coadministration of a singly labeled nuclear and singly labeled fluorescent agent containing the same targeting moiety may seem like a simpler strategy to achieve the clinical and preclinical aims described above, differences in pharmacokinetics (PK), binding affinities, physical half-life, and the complexities of co-administration of two distinct imaging agents restrict practical application. Given that the physiological properties of most NIRF dyes are based on organic molecules with molecular weight $(\mathrm{MW}) \sim 1,000 \mathrm{Da}$, while typical radioisotope chelators are polar molecules 300 $400 \mathrm{Da}$ (Fig. 3), it is highly unlikely that a singly labeled nuclear and singly labeled fluorescent agent will have comparable biodistribution and pharmacokinetic properties. Indeed, the nuclear imaging literature is replete with examples of targeting moieties that, when labeled with different chelators and radioisotopes, exhibit differing biodistribution and PK that can impact imaging performance [9-12].

While NIRF molecular imaging has demonstrated high sensitivity, when NIRF dyes are incorporated in a duallabeled format, their performance will depend critically upon the stability and optical properties of the fluorescent dye after subjection to conditions of radiolabeling. Since most dual-labeled agents under development undergo radiolabeling in the final step, factors such as radiolabeling conditions, radiolysis, or interaction between radiometal and fluorophore must be considered. Thus, there is an urgent need for characterizing optical properties of NIRF dyes after radiolabeling to determine dual-labeling combinations that are likely to best retain the fluorescence intensity of NIRF dyes. The recent surge of commercial NIRF dye development with favorable optical properties, enhanced stability, and different reactive groups for conjugation now afford numerous opportunities for probe development, but there are limited reports 
a

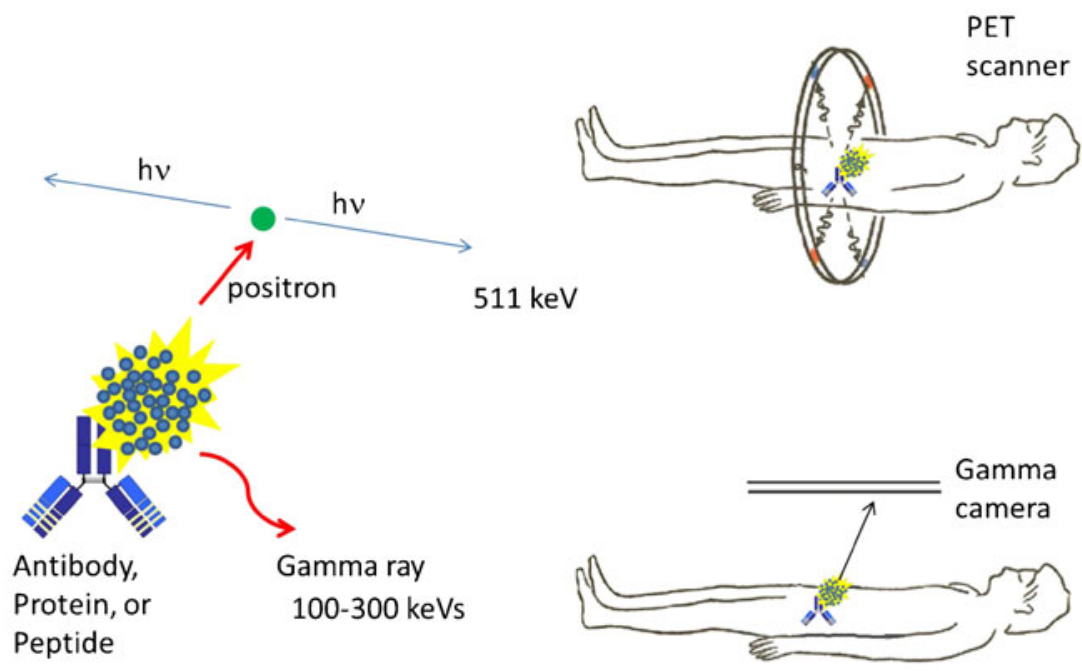

b
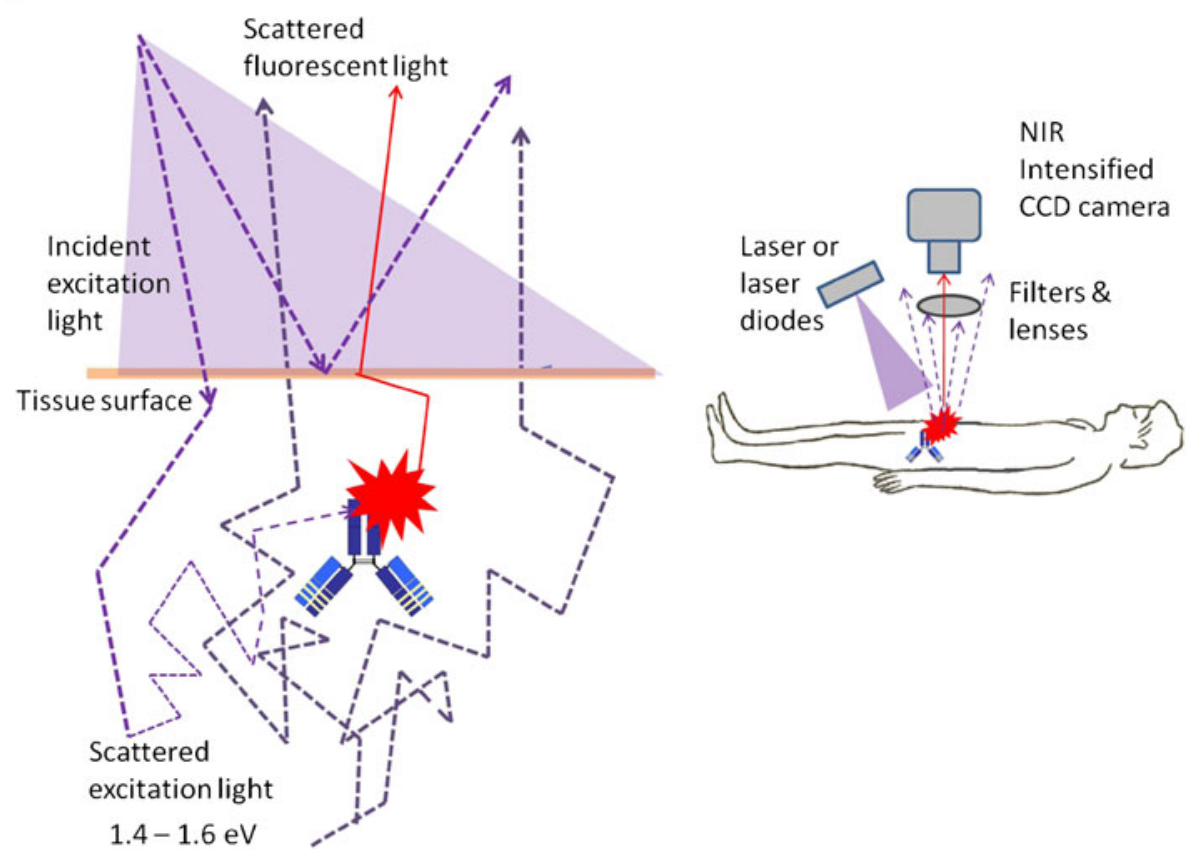

Fig. 2. a Schematic representing the physics of nuclear imaging modalities whereby upon radiative decay, high-energy photons are emitted and detected with limited scattering effects. b Schematic representing the physics of NIRF imaging whereby upon activation of the fluorophore by excitation light, the emitted fluorescence signal is highly scattered in tissue prior to detection by a CCD-based camera system.

which compare their optical properties under conditions for dual labeling. With the convergence of diverse radiochemistry formulations for key radiometals employed in nuclear imaging, limited assessment of optical properties for dual-labeled probes and variable sensitivity between existing NIRF imaging systems, it is conceivable that current radiolabeling approaches may adversely affect fluorescent properties and reduce the effectiveness of a NIRF dye as a contrast agent. Recent reviews have addressed dual-modality probes with various noninvasive imaging platforms [13, 14] and multimodal tumortargeting peptides with fluorescent and NIRF dyes [15]; however, to date, there is a paucity of information available in the literature to guide researchers in dual-labeling schemes that would preserve NIRF sensitivity.

In this review, we examine the compositions and characterization of dual-labeled imaging agents and focus on the development of chelator-based monoclonal antibody $(\mathrm{mAb})$ and peptide-targeting moieties. Herein, we limit our analysis to dual-labeled probes possessing organic NIRF dyes which emit at 750-900 nm, as these have agents have the greatest potential for clinical translation, offer the lowest background due to the lack of autofluorescence at excitation wavelengths $>750 \mathrm{~nm}$, and consequently possess the greatest sensitivity $[7,16]$. Also, based on our ongoing research with 
<smiles>O=C(O)CN(CCN(CC(=O)O)CC(=O)O)CC(=O)O</smiles>

DTPA

(393.35)

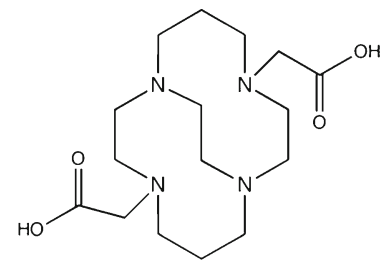

CB-TE2A

(342.43)

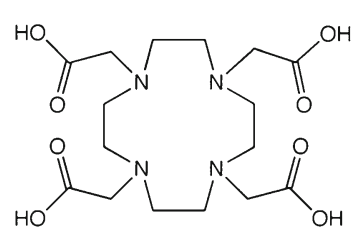

DOTA

(404.42)

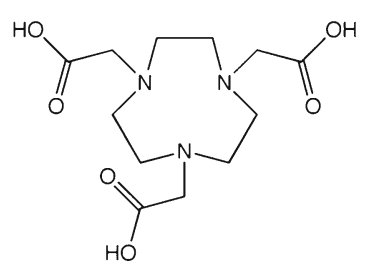

NOTA

(303.31)
Fig. 3. Examples of BFCAs used for radiolabeling (MW shown).

novel fluorophores and imaging conjugates, we present new data on the optical properties of several commercially available NIRF dyes. Specifically, we conducted a systematic study on the NIRF dyes listed in Table 1 to directly compare their optical properties in (1) water, (2) nonradioactive labeling conditions, and (3) conventional radiolabeling. We selected buffering schemes based on the most frequently employed radiometals in dual-labeled probe development, technetium-99m $\left({ }^{99 \mathrm{~m}} \mathrm{Tc}\right)$, indium-111 $\left({ }^{111} \mathrm{In}\right)$, copper-64 $\left({ }^{64} \mathrm{Cu}\right)$, and gallium-68 $\left({ }^{68} \mathrm{Ga}\right)$, to ensure relevance of the findings for both ongoing preclinical research and future translational opportunities. Ultimately, we provide perspectives on the opportunities for multimodal imaging in preclinical and translational research.

\section{Dual-Labeled Imaging Agents-mAb-Based Targeting}

Full length $\mathrm{mAbs}$ and $\mathrm{mAb}$ fragments offer an excellent approach for creating high-affinity, dual-labeling imaging agents because of (1) the high specificity for targets, (2) an abundance of reactive amino acid residues (i.e., lysines and cysteines) for conjugation of chelators and NIRF dyes, (3) the plethora of therapeutic mAbs on the market and within clinical trials, (4) the clinical acceptance of radioimmunoscintigraphy, and (5) the versatility of imaging agent design offered by $\mathrm{mAb}$ engineering [17]. Table 2 lists the published studies that utilize existing therapeutic monoclonal mAbs for preclinical imaging of human epidermal growth factor receptor-1 and 2 (HER-1, HER-2), epidermal growth factor receptor-1 (EGFR), CD20, and epithelial cell adhesion molecule (EpCAM), and identifies the different duallabeling strategies and chemical compositions (i.e., selection of chelator, NIRF dye, radiochemistry scheme, optical characterization) employed.

\section{Single-Photon-Emitting Agents}

Trastuzumab is a therapeutic agent that targets HER-2, a disease marker that is conserved in metastases in $30 \%$ of all breast cancers [18] and is increasingly associated with other cancers [19-22]. In their studies of NIRF and SPECT imaging of duallabeled trastuzumab in a xenograft model of breast cancer, Sampath et al. prepared a multimodal mAb using IRDye $800 \mathrm{CW}$ and diethylenetriaminepentaacetic acid (DTPA) for ${ }^{111}$ In radiolabeling [23]. Their findings showed that planar NIRF images possessed similar target-to-background ratios, but reduced noise when compared to SPECT images. Their superior imaging qualities in the shallow tissues of animals may be a result of the higher photon count rate of NIRF as opposed to radioactive labels. Ogawa and colleagues targeted HER-1 and HER-2 in an attempt to take advantage of the mutual cooperation of nuclear and NIRF imaging through the development of a quantitative radiolabeled probe and an activatable optical probe [24]. The authors used mAbs dual-labeled with ${ }^{111}$ In-DTPA and an ICG analog that possessed a reactive group for bioconjugation. In vitro microscopy demonstrated that there was no fluorescence signal with either conjugate when the probes were bound to cell surface antigens. After internalization, bright fluorescence signal was detectable only in the target cells, demonstrating that both conjugates work as activatable probes. The findings were confirmed by in vivo optical imaging and similar biodistribution profiles were obtained with radio and optical dual-labeled probes. In a study by Aldrich et al., trastuzumab was dual-labeled with IRDye $800 \mathrm{CW}$ and ${ }^{111} \mathrm{In}$ DTPA to establish a novel, validated assay to assess the amount of free dye in solution [25]. The authors found that the sodium dodecyl sulfate polyacrylamide gel electrophoresis (SDSPAGE) method was accurate and valid for free dye detection with applicability for other NIRF containing $\mathrm{mAb}$ imaging agents where high-performance liquid chromatography (HPLC) assessment of purity is not feasible.

Cetuximab targets EGFR overexpressed in metastatic cancers, and is a therapeutic in metastatic colorectal and head and neck cancers. Xu et al. developed dual-labeled analogs of trastuzumab and cetuximab with $\mathrm{Cy} 7$ and ${ }^{111} \mathrm{In}$ DTPA through a novel modular synthetic strategy resulting in mAb-based imaging probe libraries [26]. They showed strong binding to cancer cells and described potential use of their strategy for other targeting vectors (e.g., mAbs, affibodies, and peptides) through thiol-based linkage.

\section{PET Agents}

All of the dual-labeled mAbs reported above underwent SPECT imaging in part due to the long half-life of ${ }^{111}$ In and its well-known chelation chemistry with DTPA. The use of PET offers several advantages over planar scintigraphy and SPECT including higher sensitivity ( $2-3$ orders of magnitude) and better spatial resolution, and is increasingly used in the clinic for the diagnosis, staging, and restaging of malignant 


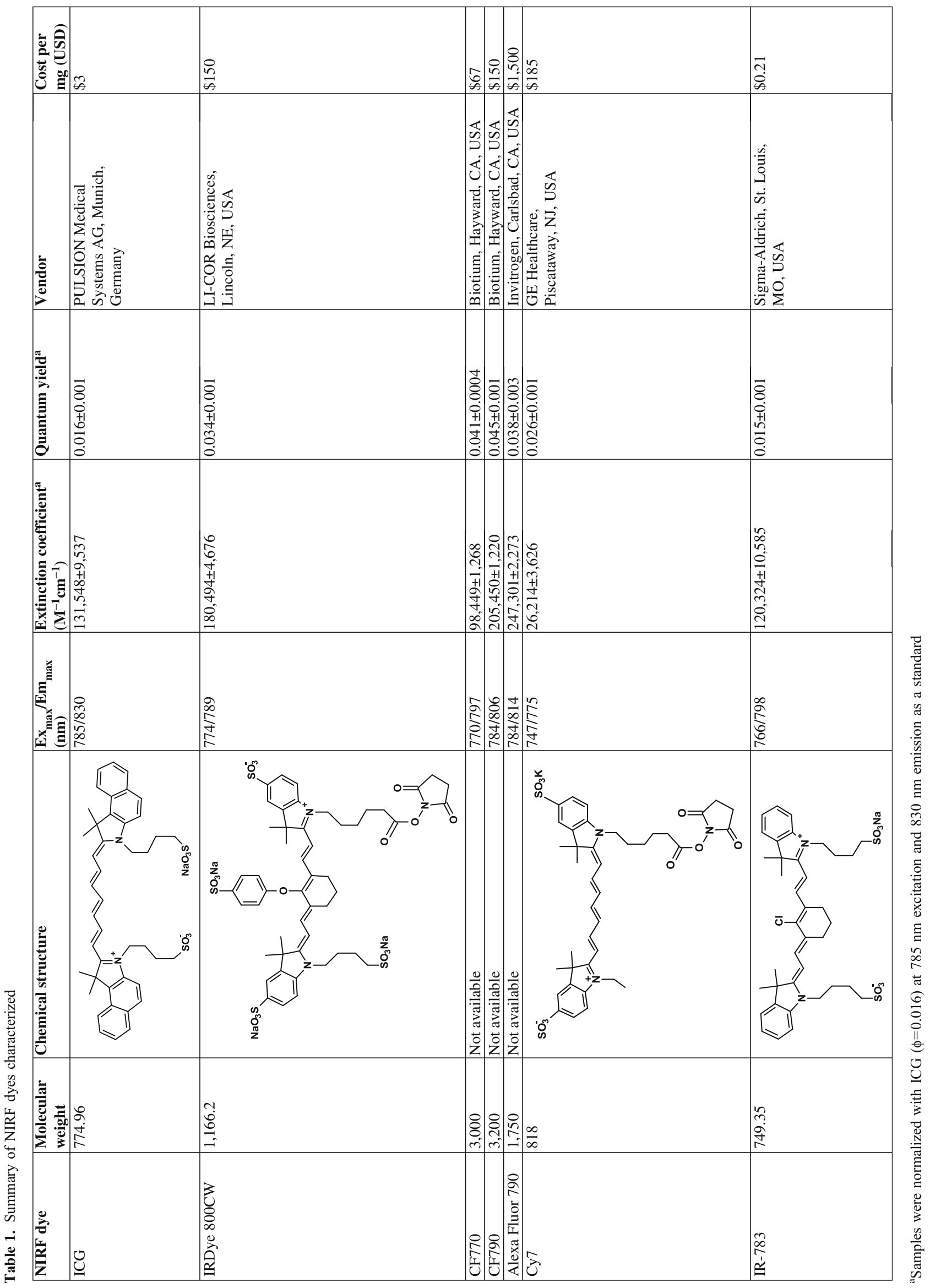




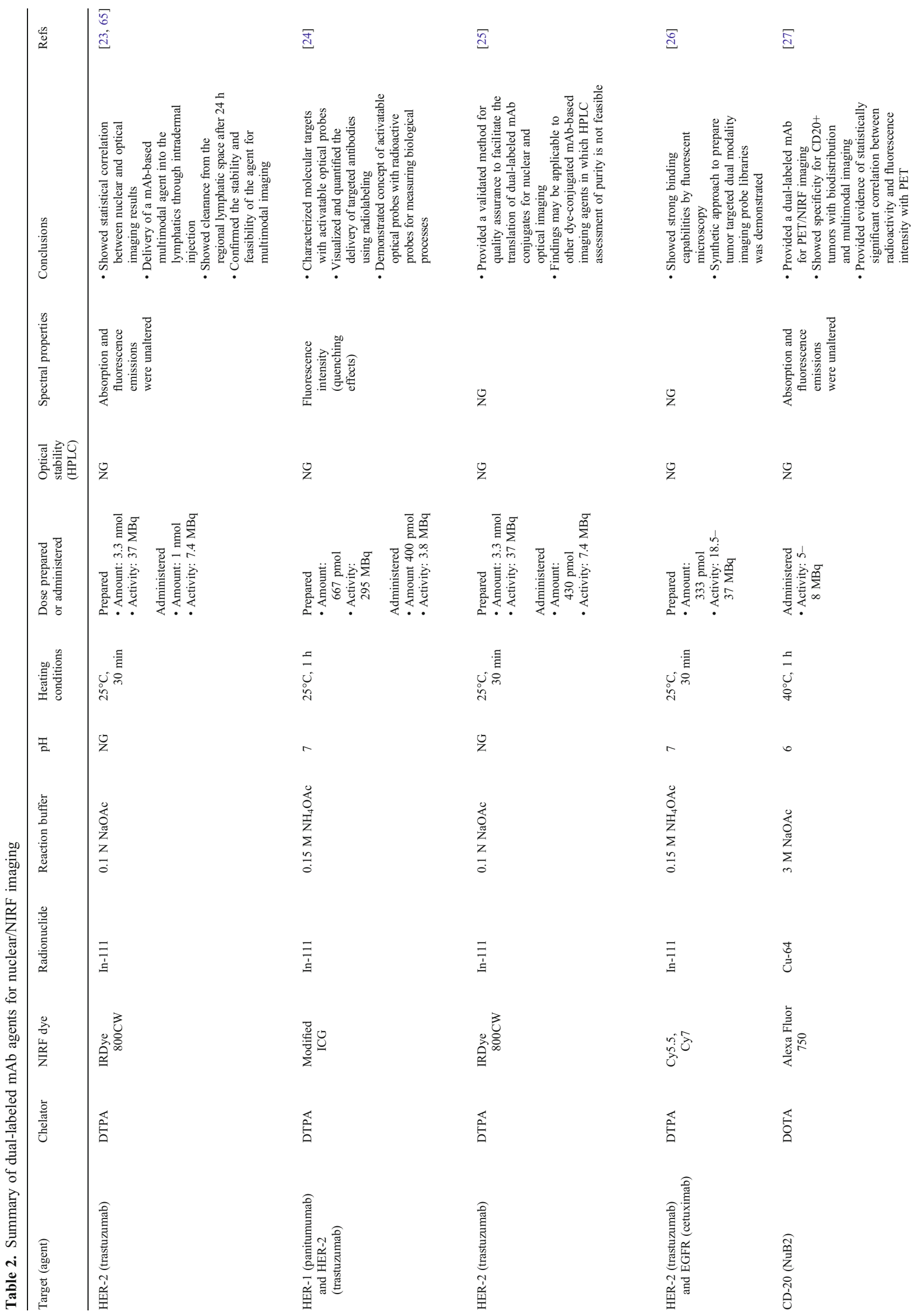




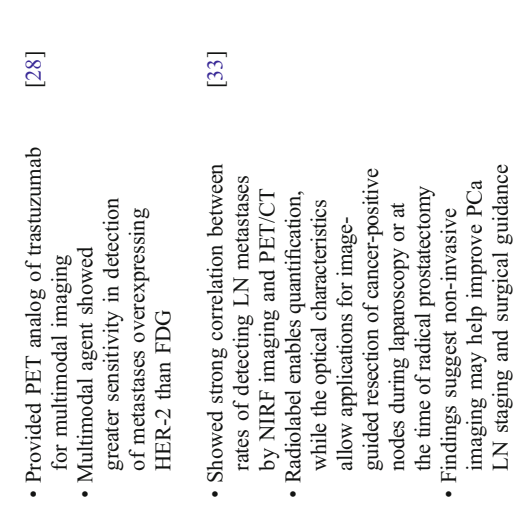

そ

¿
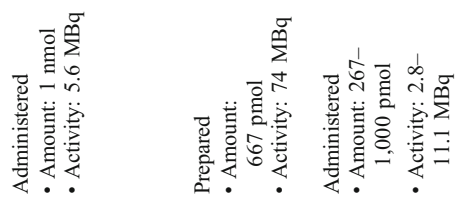

$=$
Un
0
0

in

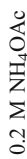

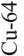

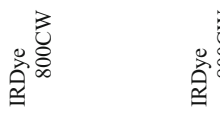

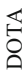

I
ப́
0
\&

范

そ

\section{そ}

6

0
0
Z
$z$
$z$

$\int_{\substack{1 \\ 1}}^{1}$

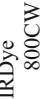

diseases. The first published demonstration of a multimodal $\mathrm{mAb}$ agent to that utilizes the PET platform was by Paudyal et al. for imaging CD20 lymphoma in mice [27]. Anti-CD20 mAbs have become the mainstay in the treatment of nonHodgkin's lymphomas and have shown efficacy in patients with B-cell chronic lymphocytic leukemia. The authors used the anti-CD20 murine mAb NuB2 for targeting CD20 positive tumors and used the positron emitting radionuclide, ${ }^{64} \mathrm{Cu}$, through the use of 1,4,7,10-tetraazacyclododecane-1,4,7,10tetraacetic acid (DOTA) as the bifunctional chelating agent (BFCA), along with Alexa Fluor 750. They were able to show that ${ }^{64} \mathrm{Cu}$-DOTA-NuB2-Alexa Fluor 750 exhibits strong and specific binding to the tumor-expressing CD20 antigen through ex vivo biodistribution and in vivo $\mathrm{PET}$ and NIRF imaging. In addition, there was a statistically significant correlation between radioactivity and fluorescence intensity, confirming the stability of the dual functional probe.

Another example of a dual-labeled mAb used for PET was given by Sampath and colleagues who developed a dual-labeled analog of trastuzumab for PET/NIRF imaging to detect metastatic breast cancer [28]. The authors conjugated ${ }^{64} \mathrm{Cu}$-DOTA and IRDye $800 \mathrm{CW}$ to the $\mathrm{mAb}$ and their findings showed excellent tumor localization which benefitted from the increased resolution of PET compared to the previous trastuzumab reports using ${ }^{111}$ In-SPECT. Also, the use of PET imaging permitted improved quantification of tracer uptake in local and metastatic sites.

Further use of PET imaging for multimodal probe development was shown in a study targeting EpCAM expression in prostate cancer. Therapeutic mAbs against EpCAM are currently in several clinical trials for the indication of preventing metastases in patients diagnosed with various cancers [29] and are used diagnostically for detection of circulating tumor cells [30-32]. In a study by Hall and colleagues, mAbs against EpCAM were dual-labeled with IRDye $800 \mathrm{CW}$ and ${ }^{64} \mathrm{Cu}$-DOTA and provided both intraoperative and non-invasive imaging of cancer-positive LNs in an orthotopic model of metastatic human prostate cancer [33]. The authors found that NIRF planar imaging possessed greater sensitivity and specificity for tumor-positive lesions than PET imaging. Although the performance of planar imaging is not directly comparable to tomographic imaging, these results nonetheless provide evidence of the added utility that planar NIRF imaging could provide to nuclear imaging modalities, especially in intraoperative guidance.

\section{Dual-Labeled Imaging Agents-Peptide-Based Targeting}

The growing interest in multimodal imaging has relied heavily upon the robustness of peptide chemistry to extend the role of existing radiopeptides to carry both a BFCA and NIRF dye. The fact that the great majority of dual-labeled imaging agents reported in the literature is peptide-based attests to the versatility of peptide chemistry as a manufacturing platform for probe development. Table 3 provides a 


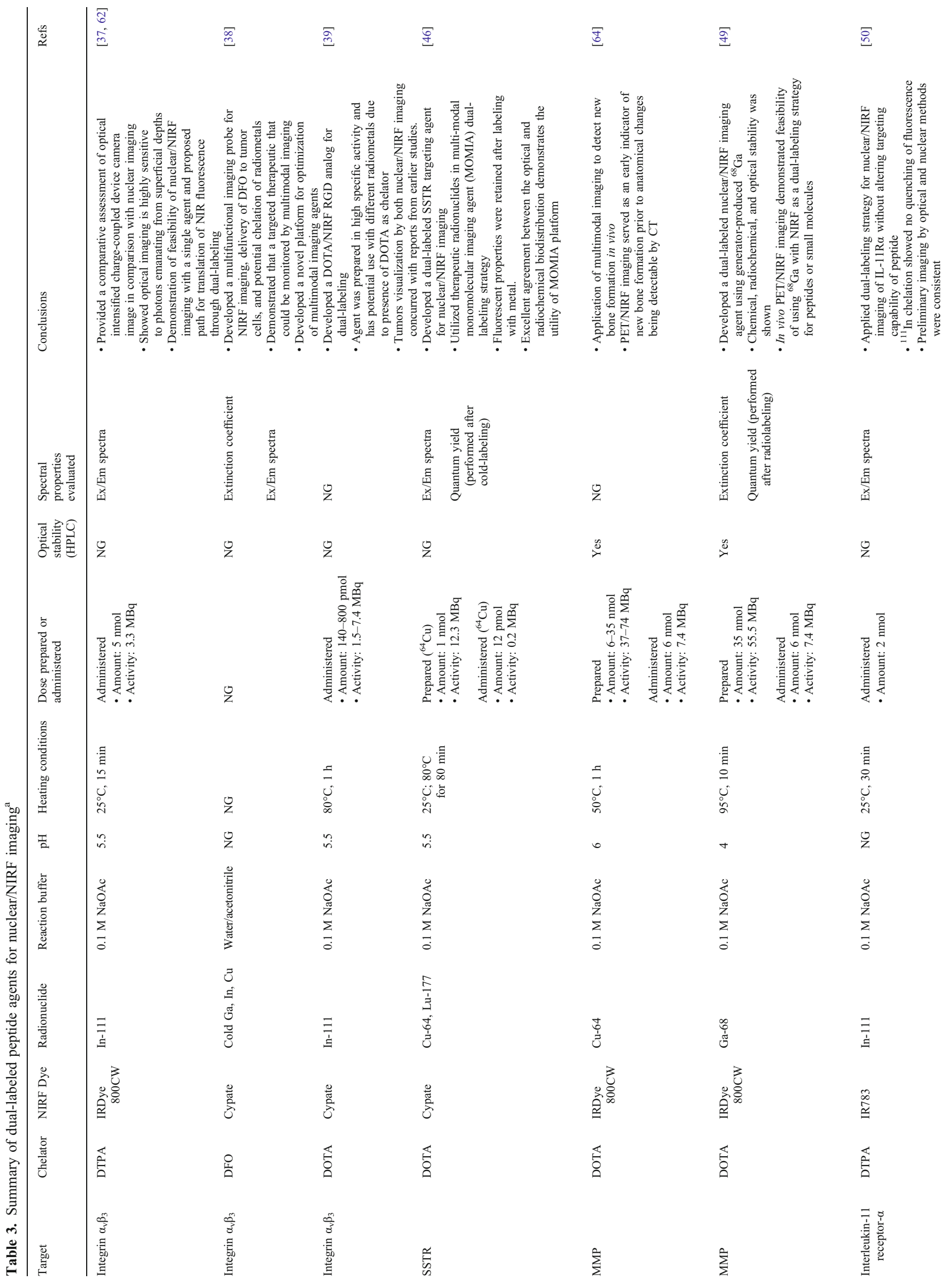




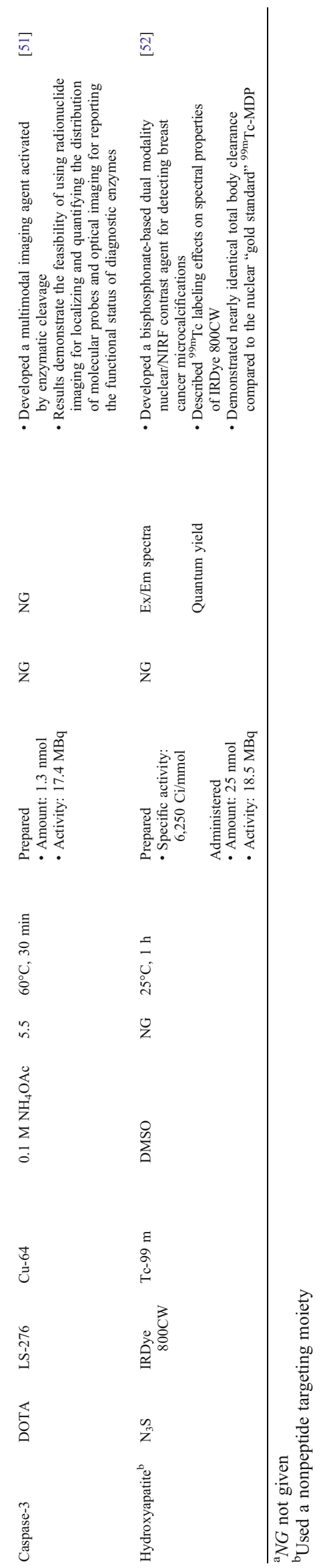

listing of the dual-labeled peptide tracers that emit in the NIR wavelength range of 750-900 $\mathrm{nm}$ and have been investigated preclinically. Predominantly, dual-labeling efforts build upon known radiotracers by utilizing established molecular targeting moieties, BFCAs, and radiolabeling schemes, and appending these with NIRF dyes. The majority of targets evaluated are based on wellcharacterized radiopeptides that target $\alpha_{\mathrm{v}} \beta_{3}$ integrins, SSTRs, and matrix metalloproteinases (MMPs) [34-36].

The dependence of solid tumors on increased blood supply through new vessel formation has been studied noninvasively with the $\alpha_{\mathrm{v}} \beta_{3}$ integrin-targeting peptide sequence Arg-Gly-Asp (RGD). Houston et al. prepared a dual-labeled RGD agent using DTPA, IRDye $800 \mathrm{CW}$, and ${ }^{111} \mathrm{In}$ and demonstrated the feasibility of combined nuclear/NIRF imaging following administration of a dual-labeled agent [37]. They also performed image quality assessment of an optical intensified charge-coupled device-based camera imaging in comparison with nuclear imaging. Their findings demonstrated an approach for cross-validating NIRF imaging platforms with nuclear imaging, an essential component for clinical translation of functional planar NIR imaging that may be achieved through dual-labeling strategies. Ye et al. developed a multifunctional RGD imaging probe for NIRF imaging, delivery of desferrioxamine to tumor cells, and potential chelation of radiometals [38]. They provided evidence of a targeted therapeutic that could be monitored by multimodal imaging and in doing so, developed a novel platform for optimization of multimodal imaging agents. In a study by Edwards et al., the RGD peptide was conjugated with cypate and DOTA, and radiolabeled with ${ }^{111}$ In with high-specific activity for SPECT imaging [39]. They prepared a DOTA/NIRF RGD analog for dual-labeling which has potential use with different radiometals due to presence of DOTA as the chelator. The authors also reported high binding affinity for the $\alpha_{\mathrm{v}} \beta_{3}$ integrin with receptorspecific binding in vitro, along with excellent agreement between the optical and nuclear imaging results in mice.

The SSTR has been actively investigated in the imaging field due to its overexpression in several types of neuroendocrine tumors and potential as a biomarker for diagnosis and therapy. Discovery of the receptor antagonist octreotide [40], and its subsequent development into an imaging agent, has been used for SPECT [41, 42] and PET imaging [43-45] of NETs. Edwards and colleagues prepared a dual-labeled SSTR agent for PET/NIRF imaging by attaching the NIRF dye cypate to the C-terminus of DOTA-octreotate and radiolabeling with ${ }^{64} \mathrm{Cu}$ [46]. Their agent for nuclear/NIRF imaging was developed alongside efforts to utilize therapeutic radionuclides in a multimodal monomolecular imaging agent (MOMIA) dual-labeling strategy. Characterization of spectral properties showed that fluorescent properties were retained after labeling with metal. Although the in vivo images did not reflect the in vitro results, the authors did observe excellent agreement between the optical and radiochemical biodistribution showing the promise of the MOMIA platform. 
Expression of MMP-9 during remodeling of the extracellular matrix has been shown to be a potential biomarker for heterotopic ossification [47]. Rodenberg et al. developed an MMP-9-targeting peptide conjugated with DOTA and IRDye $800 \mathrm{CW}$, followed by labeling with ${ }^{64} \mathrm{Cu}$ for early detection of new bone formation by PET [48]. This study demonstrated the application of multimodal imaging to detect new bone formation in vivo and demonstrated the utility of PET/NIRF imaging for early detection of molecular changes prior to anatomical changes detected by computed tomography. A follow-up study using the same bioconjugate resulted in a dual-labeled nuclear/NIRF imaging agent with generator-produced ${ }^{68} \mathrm{Ga}$ and provided detailed characterization of chemical, radiochemical, and optical properties of a dual-labeled imaging agent [49]. Multimodal imaging demonstrated the feasibility of using ${ }^{68} \mathrm{Ga}$ with NIRF as a dual-labeling strategy for peptides or small molecules, though modifications to improve bioactivity of the agent are needed.

Several other targets have been evaluated with multimodal peptide agents. An interleukin-11 receptor- $\alpha$ targeting peptide was dual-labeled using an ${ }^{111}$ In-DTPA chelate and IR-783 [50]. The authors reported no quenching of fluorescence after radiolabeling, demonstrating a promising approach for SPECT/NIRF imaging. In a study by Lee et al., multimodal imaging of caspase-3 activity was conducted by using a combined activatable and radiolabeled multimodality molecular probe, demonstrating potential uses of multimodal imaging agents activated by enzymatic cleavage [51]. Although the targeting agent was not peptide-based, it is noteworthy to include a study where a SPECT/NIRF probe based on bisphosphonate was used to detect breast cancer microcalcifications [52]. ${ }^{99 \mathrm{~m}} \mathrm{Tc}$ labeling effects on the spectral properties of IRDye $800 \mathrm{CW}$ were assessed and the authors showed promising results which demonstrated nearly identical total body clearance compared to the nuclear "gold standard" ${ }^{99 \mathrm{~m}} \mathrm{Tc}-\mathrm{MDP}$.

With the exception of the ${ }^{68} \mathrm{Ga}$ MMP-9 targeting probe [49], dual-labeled peptide imaging agents have used longerlived radiometals with half-lives ranging from $6 \mathrm{~h}$ to nearly 1 week. This approach is effective in the early stages of agent development to (1) enable longitudinal imaging at early and delayed time points, (2) monitor biodistribution, clearance routes, agent stability, and (3) determine the optimal time point for imaging; however, it does not take full advantage of the favorable PK properties of peptides for early (i.e., $1-2 \mathrm{~h}$ ) imaging. Direct comparison of a dual-labeled MMP peptide with ${ }^{64} \mathrm{Cu}$ and ${ }^{68} \mathrm{Ga}$ showed faster clearance of the ${ }^{68} \mathrm{Ga}$ complex from blood and other background tissues, enabling imaging at $1 \mathrm{~h}$. Thus, further examination of ${ }^{68} \mathrm{Ga}$ analogs for dual labeling is warranted and may offer an efficient route for agent development and pharmacological evaluation.

\section{Significance of Characterizing Optical Properties}

In order to maximize the performance of dual-labeled conjugates for multimodality imaging, the selection of an optimal radionuclide/dye combination is required. Moreover, characterization of dye performance in response to radiolabeling would help guide efficient development of novel duallabeled imaging probes and help expand the utility of nuclear imaging agents to include intraoperative guidance. Yet despite the progress on dual-labeled agent development, there has been little effort to catalog the fluorescence intensity of NIRF dyes under the various radiolabeling conditions. Such characterization is performed by evaluation of excitation/emission spectra, absorption cross section, quantum efficiency, and in some cases, fluorescent lifetime. Spectral analyses have primarily been reported on dual conjugates (i.e., agent with NIRF dye and BFCA, but no radiometal), but neglect the effects of metals (or radiometals) on optical properties.

Although the majority of dual-labeling approaches exhibit some overlap with regard to selection of NIRF dyes, BFCAs, and radiometals, to date there are no standardized methods for assessing product quality for a dual-labeled agent. Table 4 shows suggested characterization steps that can be employed. A strategy to evaluate the chemical integrity of the agent by HPLC, along with detailed analysis of the spectral properties by fluorometry before and after radiolabeling, would give a good indication of the overall stability of the agent and potentially establish specifications that could be used for translational studies. Such analyses could be performed before and after radiolabeling for comparative purposes in addition to standard quality control experiments for radiotracers such as radio-thin-layer chromatography (radio-TLC) or radio-HPLC. In the case of $\mathrm{mAbs}$, the randomness of the conjugation reactions necessitates additional characterization to determine (1) immunoreactivity

Table 4. Analytical methods for characterizing dual-labeled agents

\begin{tabular}{|c|c|c|}
\hline \multirow[t]{2}{*}{ Assay type } & \multicolumn{2}{|l|}{ Targeting vector } \\
\hline & Peptide-based & mAb-based \\
\hline \multicolumn{3}{|l|}{ Chemical } \\
\hline \multirow{4}{*}{$\begin{array}{l}\text { Characterization/ } \\
\text { purification }\end{array}$} & RP-HPLC & SEC-HPLC \\
\hline & Mass spectrometry & Mass spectrometry \\
\hline & NMR & SDS-PAGE \\
\hline & Stability studies & Stability studies \\
\hline \multicolumn{3}{|l|}{ Biological } \\
\hline Characterization & $\begin{array}{l}\text { Confirm bioactivity/ } \\
\text { affinity }\end{array}$ & $\begin{array}{l}\text { Assess } \\
\quad \text { immunoreactivity }\end{array}$ \\
\hline \multicolumn{3}{|l|}{ Radiochemical } \\
\hline \multirow[t]{5}{*}{ Characterization/purification } & RP-HPLC & SEC-HPLC \\
\hline & Radio-TLC & Radio-TLC \\
\hline & $\begin{array}{l}\text { Solid-phase } \\
\quad \text { extraction (RP) }\end{array}$ & SEC columns \\
\hline & & $\begin{array}{l}\text { Chelators } \\
\text { quantification }\end{array}$ \\
\hline & & Radiometal quenchers \\
\hline \multicolumn{3}{|l|}{ Optical } \\
\hline \multirow{2}{*}{ Characterization } & RP-HPLC & SEC-HPLC \\
\hline & Spectral properties & $\begin{array}{l}\text { Spectral properties } \\
\text { Dye/protein ratio }\end{array}$ \\
\hline
\end{tabular}

$R P-H P L C$ reversed phase high-performance liquid chromatography, $S E C-H P L C$ size-exclusion reversed phase high-performance liquid chromatography, $N M R$ nuclear magnetic resonance, radio-TLC radio-thinlayer chromatography, $S D S-P A G E$ sodium dodecyl sulfate polyacrylamide gel electrophoresis 
by a modified Lindmo assay [33] or NIR-based flow cytometry, (2) dye/protein ratio by spectroscopy, and (3) BFCA/protein ratio using a carrier-added radiolabeling method [53].

The lack of validating NIRF dye stability and optical properties under radiolabeling conditions may be attributed to failed, first clinical studies which combined ${ }^{99 \mathrm{~m}} \mathrm{Tc}$ and ICG for LN staging. In a study of sentinel LN mapping in breast cancer by Sevick-Muraca et al., HPLC analysis of ICG following addition to pertechnetate showed instability of the dye, resulting in a loss of fluorescence optical properties [6] due presumably to the instability of ICG at lower pH [54]. While gamma scintigraphy and blue dye (but not NIRF) enabled efficient detection of sentinel LNs, it was only when ICG was injected separately that LNs and lymphatic structures were readily visualized by NIRF. Recently, Buckle et al. reported on a multimodal formulation using albumin colloids that sequester ICG in high concentrations and likely prevent the stacking of molecules and subsequent quenching of the dye, ultimately resulting in more stable complexes with higher target-tobackground ratios [55]. While addressing the quenching issues, the effects of $\mathrm{pH}$ and radiolabeling formulation on the optical properties of ICG have yet be determined for the nanocolloid formulation and are warranted given the known stability concerns with ICG. Nonetheless, the formulation was evaluated in patients and demonstrated the effectiveness of a hybrid multimodal agent which combined fluorescent ICG and human serum albumin-based ${ }^{99 \mathrm{~m}} \mathrm{Tc}$-radiocolloid [56]. Continued efforts to improve the formulation of dual-labeled agents are needed to conserve (or enhance) NIRF properties, permit highly sensitive detection of the agent following microdose administration, and facilitate their translation into clinical practice.

\section{Comparison of NIRF Dyes under Dual-Labeling Conditions}

\section{Spectral Properties in Nonradioactive Labeling Schemes}

The commercially available NIRF dyes listed in Table 1 were obtained as lyophilized powders and stored at $-20^{\circ} \mathrm{C}$ with protection from light. Nonradioactive-labeling conditions were selected based on the conditions in Table 5 as follows: (1) ${ }^{99 \mathrm{~m}} \mathrm{Tc}$ : PBS, $\mathrm{pH} 7.4$, add $50 \mu \mathrm{L}$ of $\mathrm{SnCl}_{2}(2 \mathrm{mg} / \mathrm{ml}$ $0.05 \mathrm{M} \mathrm{HCl}) ;(2){ }^{64} \mathrm{Cu}: 0.1 \mathrm{M} \mathrm{NaOAc}$, pH 6; (3) ${ }^{111} \mathrm{In}: 0.2 \mathrm{M}$ $\mathrm{NaOAc}, \mathrm{pH}$ 6; and (4) ${ }^{68} \mathrm{Ga}: 1.25 \mathrm{M} \mathrm{NaOAc}, \mathrm{pH} 4$. Figure 4 shows the experimental design used. All solutions were kept at room temperature and evaluated within $1 \mathrm{~h}$ of preparation.

\section{Preparation of Radioactive Solutions}

Dyes which showed greater fluorescence intensity (i.e., extinction coefficient (EC), QY) than ICG proceeded to testing with radioactive solutions. ${ }^{64} \mathrm{CuCl}_{2}$ was obtained from Washington University (St. Louis, MO, USA) and diluted with $0.1 \mathrm{~N} \mathrm{NaOAc}(\mathrm{pH}$ 6). Radioactivity (11.1 MBq) was added to solutions of dye in the same buffer with a final volume of $300 \mu \mathrm{L}$. Reactions were heated at $50^{\circ} \mathrm{C}$ for $30 \mathrm{~min}$ followed by dilution to a $6 \mu \mathrm{M}$ final concentration with $10-15 \mathrm{~mL}$ water. Samples were used within $1 \mathrm{~h}$ for optical property assessment.

To test the effect of ${ }^{68} \mathrm{Ga}$ on optical properties, lyophilized dyes were reconstituted in $200 \mu \mathrm{L} 1.25 \mathrm{M} \mathrm{NaOAc}$ at $\mathrm{pH}$ 4. ${ }^{68} \mathrm{Ga}$ was eluted in-house from a commercially available ${ }^{68} \mathrm{Ge} /{ }^{68} \mathrm{Ga}$ generator (Eckert \& Ziegler, Berlin, Germany) as previously described [49]. To each dye solution, increasing activities of ${ }^{68} \mathrm{Ga}(22.2,59$, and $152 \mathrm{MBq})$ were added in eluate volumes of $0.2,0.5$, and $1.2 \mathrm{~mL}$, respectively. Samples were heated at $95^{\circ} \mathrm{C}$ for 10 min to simulate ${ }^{68} \mathrm{Ga}$ labeling conditions commonly used for peptide tracers. Samples were diluted to $6 \mu \mathrm{M}$ with water or $1.25 \mathrm{M} \mathrm{NaOAc}$ (pH 4 ) and used within $1 \mathrm{~h}$ for optical property assessment.

\section{Assessment of Spectral and Chemical Properties}

Absorbance of all solutions was measured at $785 \mathrm{~nm}$ (DU800 Spectrophotometer, Beckman Coulter, Brea, CA, USA) to obtain an extinction cross section from the slope of absorbance versus dye concentration. The fluorescent properties of the solutions were evaluated using a Fluorolog Tau-3

Table 5. Conditions for conventional radiometal chelation schemes

\begin{tabular}{llll}
\hline Radiometal/chelators & Reaction buffer & $\mathrm{pH}$ & Heating temperature $\left({ }^{\circ} \mathrm{C}\right)$ \\
\hline Tc-99 m & & & $2-8$ \\
$\mathrm{~N}_{\mathrm{x}} \mathrm{S}_{4-\mathrm{x}}$ & Saline & 7.4 & 25 \\
$\quad$ Organometallic tricarbonyl & Saline & $5.5-6.5$ & $25-50$ \\
Cu-64 & $0.1 \mathrm{M}$ acetate & 5.5 & 25 \\
DOTA & $0.1 \mathrm{M}$ acetate & 8.0 & 95 \\
TETA & $0.1 \mathrm{M}$ acetate & $6-8$ & 25 \\
CB-TE2A & $0.1 \mathrm{M}$ acetate & $5-6$ & 25 \\
NODAGA & $0.1-1.0 \mathrm{M}$ acetate & $25-95$ \\
In-111 & $0.1-1.0 \mathrm{M}$ acetate & $5-6$ & 95 \\
DTPA & $0.1-1.25 \mathrm{M}$ acetate & $3.0-4.5$ & $25-40$ \\
DOTA & $0.1-1.0 \mathrm{M}$ acetate & $3.5-4.5$ & \\
Ga-68 & & & \\
DOTA & & \\
NOTA, NODAGA & & \\
\hline
\end{tabular}


Expt \#1: water as solvent

- Spectral properties (Fluorolog)

- Chemical stability (HPLC)

Expt \#3: Effects of radioactivity

${ }^{68} \mathrm{Ga} /{ }^{64} \mathrm{Cu}$ radiolabeling

- Spectral properties (Fluorolog)

- Chemical stability (HPLC)

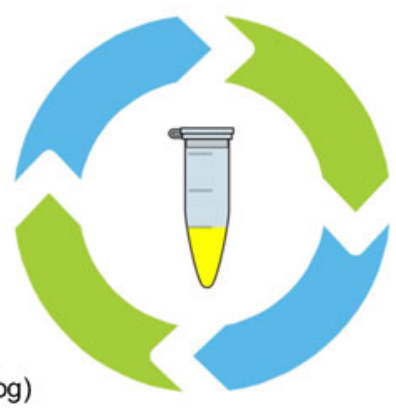

Expt \#2: non-radioactive

buffer systems as solvents

- Spectral properties (Fluorolog)

Fig. 4. Experimental design for characterization of NIRF dyes in water, buffers, and radioactive solutions.

spectrofluorometer (Horiba Jobin Yvon, Edison, NJ, USA) at $\mathrm{Ex} / \mathrm{Em}=785 / 830 \mathrm{~nm}$ to compute QY $(\phi)$ using the method of Williams et al. [57], with ICG $(\phi=0.016)$ at $E x / E m=785 /$ $830 \mathrm{~nm}$ serving as a standard [58]. Relative brightness (RB) of each NIRF dye relative to ICG was computed by dividing the product of EC and QY with that of ICG. Chemical stability of selected dye solutions (both radioactive and nonradioactive) was evaluated by analytical HPLC as previously described [49]. Stability of nonradioactive samples in water was measured at 0 , $4,6,12$, and $24 \mathrm{~h}$ after preparation, while ${ }^{68} \mathrm{Ga}$ samples were analyzed at 0,2 , and $4 \mathrm{~h}$ post-mixing. Each dye showed excellent chemical stability in water over the time points investigated as no new peaks were detected on UV $(280 \mathrm{~nm})$ or fluorescence channels. Similarly, no radiolytic or breakdown products were observed by HPLC following incubation with ${ }^{68} \mathrm{Ga}$ on UV $(280 \mathrm{~nm})$ or fluorescence channels.

\section{Roadmap for Efficient Design and Implementation of Dual-Labeled Agents}

The ability of NIRF dyes to withstand various radiolabeling conditions and maintain optical integrity is critical for the development and advancement of dual-labeled imaging agents. Comparison of several commercially available NIRF dyes in water showed that each dye, with the exception of $\mathrm{Cy} 7$ and IR783, was significantly brighter than ICG (Fig. 5). CF790 and Alexa Fluor 790 were the brightest of all dyes tested, both with a $\sim 4.5$-fold increase in RB compared to ICG. IRDye $800 \mathrm{CW}$ has been extensively used by our group and others, and showed excellent optical properties with a threefold increase in RB compared to ICG. It is noteworthy to mention that our spectral analyses were conducted using parameters optimized for ICG; thus, dyes which exhibited low fluorescence under these conditions would clearly benefit from tuning of factors such as Ex/Em wavelengths and filter settings to better demonstrate their utility in future studies.

Radiolabeling schemes incorporate a wide array of buffers, $\mathrm{pH}$ ranges, and heating temperatures, all of which can impact spectral properties of NIRF dyes. Nonradioactive radiolabeling schemes of the four most prevalent radiometals used in nuclear imaging were applied to the NIRF dyes as a preliminary step to assess potential dye/radiometal combinations. CF790 and Alexa Fluor 790 were superior under all buffer conditions tested and exhibited no significant decrease in $\mathrm{RB}$ under ${ }^{99 \mathrm{~m}} \mathrm{Tc}$ conditions (Fig. 6) compared to water controls. The harshness of reaction conditions $\left({ }^{68} \mathrm{Ga}>{ }^{111} \mathrm{In}>\right.$ ${ }^{64} \mathrm{Cu}>{ }^{99 m} \mathrm{Tc}$ ), primarily resulting from differences in $\mathrm{pH}$ and buffer concentrations, impacted RB for both CF790 and Alexa Fluor 790, while IRDye $800 \mathrm{CW}$ fluorescence remained relatively unchanged in different buffers. CF770 also showed stable fluorescent signal in each buffering system, though its RB was lower than IRDye $800 \mathrm{CW}$. As was the case with water, $\mathrm{Cy} 7$ was not as bright as ICG. The data showed distinct differences between dye performance, while also depicting certain dye/radiometal combinations that may preferentially conserve optical properties to yield more sensitive imaging agents.

The four radiometals examined have distinct radioactive emissions $\left({ }^{99 \mathrm{~m}} \mathrm{Tc}=140 \mathrm{keV} \gamma ;{ }^{64} \mathrm{Cu}=1.7 \mathrm{MeV} \beta^{+}, 578 \mathrm{keV}\right.$ $\beta^{-} ;{ }^{111} \mathrm{In}=171$ and $245 \mathrm{keV} \gamma$, Auger electrons; ${ }^{68} \mathrm{Ga}=$ 1.9 $\mathrm{MeV} \beta^{+}$) which have been exploited in biomedical applications. Spectral analysis of dye-metal complexation

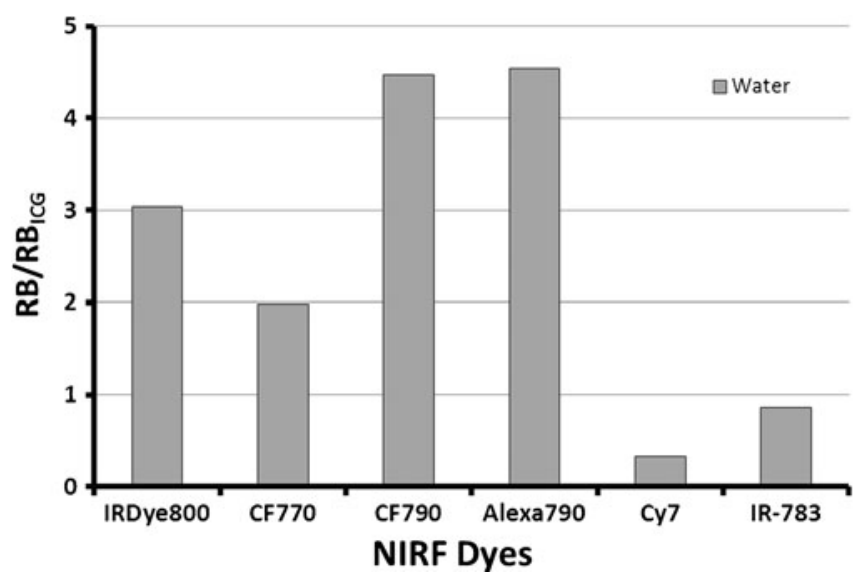

Fig. 5. Relative brightness of various NIRF dyes in water. Samples were normalized with ICG $(\phi=0.016)$ at $785 \mathrm{~nm}$ excitation and $830 \mathrm{~nm}$ emission as a standard. 


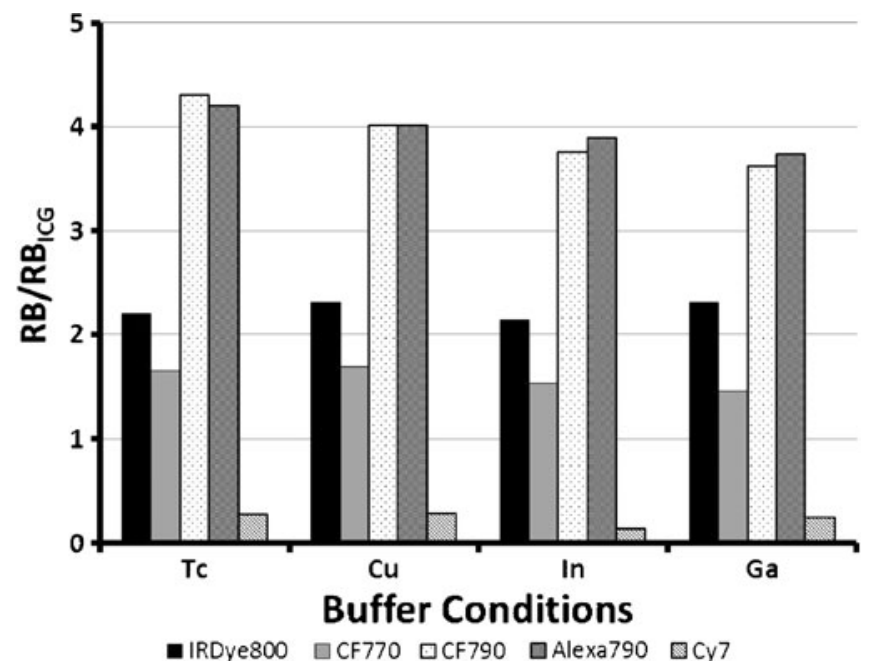

Fig. 6. Relative brightness of various NIRF dyes in buffers. Samples were normalized with ICG $(\phi=0.016)$ at $785 \mathrm{~nm}$ excitation and $830 \mathrm{~nm}$ emission as a standard.

has shown that metal ions can impact the optical stability of dual-labeled agents through the formation of charge transfer complexes between the metals and the fluorophore [59], though differences in chemical structure of dyes must be taken into account. To test the effects of clinically used radioactive metals on the NIRF dyes, we selected ${ }^{64} \mathrm{Cu}$ and ${ }^{68} \mathrm{Ga}$ due to their varying emissions, prevalence of use in molecular imaging, and the overall clinical significance of PET imaging. ${ }^{64} \mathrm{Cu}$ reduced the RB of each dye compared to water controls with varying degrees of tolerance for $0.1 \mathrm{M}$ $\mathrm{NaOAc}$ at $\mathrm{pH} 6$ and the 30 min heating step conducted at $50^{\circ} \mathrm{C}$ (RB: Alexa Fluor $790>$ CF $790>$ IRDye $800 \mathrm{CW}>$ CF770; Fig. 7). Assessment of optical properties with ${ }^{68} \mathrm{Ga}$ was performed under harsher conditions (1.25 M NaOAc at $\mathrm{pH} 4$, heating at $95^{\circ} \mathrm{C}$ for $10 \mathrm{~min}$ ) to represent a commonly

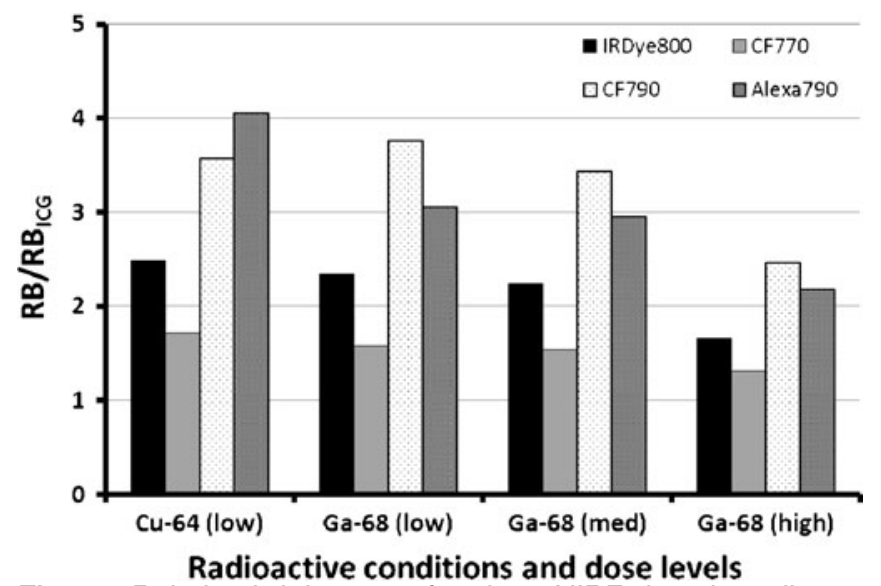

Fig. 7. Relative brightness of various NIRF dyes in radioactive solutions of ${ }^{64} \mathrm{Cu}(11.1 \mathrm{MBq})$ and ${ }^{68} \mathrm{Ga}$. Three different activity levels of ${ }^{68} \mathrm{Ga}$ were used: low $=22.2 \mathrm{MBq}$, med= $59 \mathrm{MBq}$, high=152 MBq. Samples were normalized with ICG $(\phi=0.016)$ at $785 \mathrm{~nm}$ excitation and $830 \mathrm{~nm}$ emission as a standard. used clinical formulation [60]. Also, three activity levels were examined to reflect typical preparation of a dual-labeled probe for an in vitro study (low activity, $22.2 \mathrm{MBq}$ ), an in vivo imaging study (medium activity, $59 \mathrm{MBq}$ ), and a proposed human study (high activity, $152 \mathrm{MBq}$ ). Each ${ }^{68} \mathrm{Ga}$ experiment had similar findings with the RB of CF790> Alexa Fluor 790> IRDye $800 \mathrm{CW}>\mathrm{CF} 770$ and a dose-dependent reduction in $\mathrm{RB}$ was observed.

While the empirical data provide evidence on dye performance, several other considerations must be taken into account before selecting a certain NIRF dye for agent development. First, the dyes evaluated were of varying MW, and thus can have significantly different effects on the PK profile of a multimodal agent. CF790 showed excellent optical properties under all test conditions including with ${ }^{68} \mathrm{Ga}$, making it an excellent candidate for dual-labeling; however, its size of 3,200 Da may prohibit its use with peptides due to concerns over a potential decrease in binding affinity resulting from steric effects of the bulky dye, altered biodistribution with increased blood circulation, and higher levels of hepatobiliary clearance. A more practical application of CF790 may be in its use with $\mathrm{mAbs}$ or engineered $\mathrm{mAb}$ fragments which can tolerate such an increase in MW without significant perturbation of binding, assuming the pharmacophore is not disrupted during the labeling process. Alternatively, Alexa Fluor 790 is a much smaller NIRF dye (MW 1,750 Da) and exhibited comparable optical characteristics to CF790, making it more amenable to use with peptides. A factor which may limit the use of Alexa Fluor 790 is its exorbitant cost, which is greater than tenfold higher than other dyes at the time of publication, and may serve as an obstacle when considering the future production under good manufacturing practice (GMP) required for safety and toxicity testing en route to clinical translation. Also, the chemical structures of CF770, CF790, and Alexa Fluor 790 were not readily available, with vendors only indicating that the dyes exist as NHS-esters for coupling reactions. The improved optical stability of these dyes may be attributed to the presence of modified aromatic groups, polymethine groups, or groups for improving solubility, though we can only speculate until more information on their chemical compositions are revealed. The lack of structural information also complicates agent development by limiting the ability to predict potential changes in PK which directly affect biodistribution and dosimetry measurements, criteria which need to be well-defined for translation. In contrast, IRDye $800 \mathrm{CW}$ has been well-characterized in both $\mathrm{mAb}$ and peptide-targeting schemes [23, 28, 33, 37, 61-65] and demonstrated acceptable fluorescence under all conditions. IRDye 800CW (MW 1091.1) is well-suited for peptide labeling and, with its published chemical structure, PK modulation strategies can be employed as needed into solidphase peptide synthesis to reduce potential changes resulting from increased lipophilicity or circulation time.

Ideally, a specific NIRF dye/radionuclide combination can be designated as "best" for a particular use. However, the cost of reagents, access to radioisotopes, and variability 
in imaging system infrastructure are likely to limit the practicality of such an approach within diverse research environments. Moreover, unlike full-length $\mathrm{mAbs}$ where overall agent size is quite uniform, the size of peptide-based targeting agents is highly variable and will dictate the NIRF dye selected, which then in turn impacts PK, and ultimately determines which radioisotope is best suited to obtain high signal-to-noise ratios during imaging. We envision our results serving as an impetus to stimulate characterization of the multitude of other NIRF dyes which have applications in dual-labeling. Complementary efforts by imaging scientists may lead towards the assembly of a robust database of NIRF dye/radionuclide interaction and ultimately establish a dual-labeling toolkit for smart agent design.

\section{Future Directions}

Clinical nuclear imaging stands to benefit greatly from the introduction of improved NIRF dyes. Dual-labeled agents may allow a single targeting moiety to be used throughout the patient care process for diagnosis, staging, treatment planning, surgical guidance, therapeutic monitoring, and validation of histological findings. Since molecularly targeted, chelator-based multimodal probes have yet to be used in humans, exact specifications will need to be defined for batch release and may initially follow guidelines set forth for conventional chelator-based radiotracers which are outlined in the Food and Drug Administration (FDA) guidance documents for GMP manufacturing of imaging agents [66].

Along with a better understanding of the capabilities of NIRF dyes, continued testing in preclinical studies is needed to compare the sensitivity and specificity of NIRF and nuclear imaging for potential clinical applications. The findings should build upon the existing knowledge and make significant strides towards validating multimodal imaging platforms for clinical use. Innovative chemistry strategies to combine chelators and NIRF dyes into a single "reactive" unit have been shown and offer great promise to simplify agent synthesis and broaden the use of multimodal probes [67-70]. Given the importance of mAb-based targeting approaches, such platforms may help mitigate the loss of immunoreactivity that can occur following multistep conjugations at random sites on $\mathrm{mAbs}$ or $\mathrm{mAb}$ fragments.

As dual-labeled agents proceed towards human testing, certain challenges must be addressed including detailed toxicological analysis to demonstrate safety and determine dosing capabilities. Accordingly, bulk amounts of probe will be needed for such studies, thus an efficient and scalable GMP manufacturing process will need to be developed by dye manufacturers yet still be affordable to researchers. FDA approval may be required on multiple levels as novel NIRF dyes are considered new chemical entities and will require their own rigorous preclinical characterization in addition to the requirements for NIRF dye conjugates. Reports by manufacturers such as LI-COR Biosciences indicate that
IRDye $800 \mathrm{CW}$ is navigating the regulatory process and may serve as a benchmark for other dye developers [63].

Other alternatives for dual-labeling strategies other than those described above employ isotopic substitution of a radionuclide either directly onto a NIRF dye, or on a protein, sugar, or macromolecule that is conjugated to NIRF dye. Although potentially more cumbersome than chelation chemistry, opportunities for dual-labeled agents with isotopic substitution may be possible. For example, Tsing and colleagues [71] combined a boronate trap for fluoride on an NIRF dye with an activated ester for bioconjugation. As proof of concept, the entity was conjugated to Lymphoseek, a mannosylated $16 \mathrm{kDa}$ dextran conjugate that was previously developed for radiolabeling with ${ }^{99 \mathrm{~m}} \mathrm{Tc}$ for sentinel LN mapping [72]. Facile incorporation of ${ }^{18} \mathrm{~F}$ into the NIRFlabeled Lymphoseek enables PET/NIRF imaging, yet NIRF optical properties and agent performance remain to be evaluated following radiolabeling. Given that positron annihilation requires interaction with electrons that may also be associated with the electron dense region of a fluorophore, consideration of the impact of the radioactive decay upon fluorescent properties is warranted.

The objective of this review was to provide a detailed description of the components of dual-labeled nuclear/NIRF imaging probes and evaluate combinations of dyes with key radiometals in nuclear imaging. Ultimately, imaging researchers will have the ability to select proven components from a "toolkit" for customized multimodal agent design, potentially improving imaging and translational capabilities.

Acknowledgments. This work was supported by the NIH/NCI Network for Translation Research U54-CA136404.

Conflict of Interest. The authors declare that they have no conflict of interest.

Open Access. This article is distributed under the terms of the Creative Commons Attribution Noncommercial License which permits any noncommercial use, distribution, and reproduction in any medium, provided the original author(s) and source are credited.

\section{References}

1. Te Velde EA, Veerman T, Subramaniam V, Ruers T (2010) The use of fluorescent dyes and probes in surgical oncology. Eur J Surg Oncol 36 (1):6-15

2. Marshall MV, Rasmussen JC, Tan I-CA MB, Adams KE, Wang X, Fife CE, Maus EA, Smith LA, Sevick-Muraca EM (2010) Near-infrared fluorescence imaging in humans with indocyanine green: a review and update. Open Surg Oncol J 2(1):12-25

3. Polom K, Murawa D, Rho YS, Nowaczyk P, Hunerbein M, Murawa P (2011) Current trends and emerging future of indocyanine green usage in surgery and oncology: a literature review. Cancer. doi:10.1002/cncr.26087

4. Schaafsma BE, Mieog JS, Hutteman M, van der Vorst JR, Kuppen PJ, Lowik CW, Frangioni JV, van de Velde CJ, Vahrmeijer AL (2011) The clinical use of indocyanine green as a near-infrared fluorescent contrast agent for image-guided oncologic surgery. J Surg Oncol 104(3):323-332

5. Gioux S, Choi HS, Frangioni JV (2010) Image-guided surgery using invisible near-infrared light: fundamentals of clinical translation. Mol Imaging 9(5):237-255

6. Sevick-Muraca EM, Sharma R, Rasmussen JC, Marshall MV, Wendt JA, Pham HQ, Bonefas E, Houston JP, Sampath L, Adams KE, Blanchard DK, Fisher RE, Chiang SB, Elledge R, Mawad ME (2008) Imaging of lymph flow in breast cancer patients after microdose 
administration of a near-infrared fluorophore: feasibility study. Radiology 246(3):734-741

7. Sevick-Muraca EM, Rasmussen JC (2008) Molecular imaging with optics: primer and case for near-infrared fluorescence techniques in personalized medicine. J Biomed Opt 13(4):041303

8. Culver J, Akers W, Achilefu S (2008) Multimodality molecular imaging with combined optical and SPECT/PET modalities. J Nucl Med 49 (2):169-172

9. Fani M, Del Pozzo L, Abiraj K, Mansi R, Tamma ML, Cescato R, Waser B, Weber WA, Reubi JC, Maecke HR (2011) PET of somatostatin receptor-positive tumors using $64 \mathrm{Cu}$ - and $68 \mathrm{Ga}$-somatostatin antagonists: the chelate makes the difference. J Nucl Med 52 (7): $1110-1118$

10. Rogers BE, Anderson CJ, Connett JM, Guo LW, Edwards WB, Sherman EL, Zinn KR, Welch MJ (1996) Comparison of four bifunctional chelates for radiolabeling monoclonal antibodies with copper radioisotopes: biodistribution and metabolism. Bioconjug Chem 7(4):511-522

11. Jones-Wilson TM, Deal KA, Anderson CJ, McCarthy DW, Kovacs Z, Motekaitis RJ, Sherry AD, Martell AE, Welch MJ (1998) The in vivo behavior of copper-64-labeled azamacrocyclic complexes. Nucl Med Biol 25(6):523-530

12. Dearling JL, Voss SD, Dunning P, Snay E, Fahey F, Smith SV, Huston JS, Meares CF, Treves ST, Packard AB (2011) Imaging cancer using PET - the effect of the bifunctional chelator on the biodistribution of a (64)Cu-labeled antibody. Nucl Med Biol 38(1):29-38

13. Lee S, Chen X (2009) Dual-modality probes for in vivo molecular imaging. Mol Imaging 8(2):87-100

14. Joshi BP, Wang TD (2010) Exogenous molecular probes for targeted imaging in cancer: focus on multi-modal imaging. Cancers 2(2):1251-1287

15. Kuil J, Velders AH, van Leeuwen FW (2010) Multimodal tumortargeting peptides functionalized with both a radio- and a fluorescent label. Bioconjug Chem 21(10):1709-1719

16. Adams KE, Ke S, Kwon S, Liang F, Fan Z, Lu Y, Hirschi K, Mawad ME, Barry MA, Sevick-Muraca EM (2007) Comparison of visible and near-infrared wavelength-excitable fluorescent dyes for molecular imaging of cancer. J Biomed Opt 12(2):024017

17. Olafsen T, Wu AM (2010) Antibody vectors for imaging. Semin Nucl Med 40(3):167-181

18. Tagliabue E, Balsari A, Campiglio M, Pupa SM (2010) HER2 as a target for breast cancer therapy. Expert Opin Biol Ther 10(5):711-724

19. Wilken JA, Webster KT, Maihle NJ (2010) Trastuzumab sensitizes ovarian cancer cells to EGFR-targeted therapeutics. J Ovarian Res 3:7

20. Chua TC, Merrett ND (2011) Clinicopathologic factors associated with HER2-Positive gastric cancer and its impact on survival outcomes - a systematic review. Int J Cancer. doi:10.1002/ijc.26292

21. Sorscher SM (2011) Marked response to single agent trastuzumab in a patient with metastatic HER-2 gene amplified rectal cancer. Cancer Invest 29(7):456-459

22. Tomizawa K, Suda K, Onozato R, Kosaka T, Endoh H, Sekido Y, Shigematsu H, Kuwano H, Yatabe Y, Mitsudomi T (2011) Prognostic and predictive implications of HER2/ERBB2/neu gene mutations in lung cancers. Lung Cancer 74(1):139-144

23. Sampath L, Kwon S, Ke S, Wang W, Schiff R, Mawad ME, SevickMuraca EM (2007) Dual-labeled trastuzumab-based imaging agent for the detection of human epidermal growth factor receptor 2 overexpression in breast cancer. J Nucl Med 48(9):1501-1510

24. Ogawa M, Regino CA, Seidel J, Green MV, Xi W, Williams M, Kosaka N, Choyke PL, Kobayashi H (2009) Dual-modality molecular imaging using antibodies labeled with activatable fluorescence and a radionuclide for specific and quantitative targeted cancer detection. Bioconjug Chem 20(11):2177-2184

25. Aldrich MB, Wang X, Hart A, Kwon S, Sampath L, Marshall MV, Sevick-Muraca EM (2011) Assessment of free dye in solutions of duallabeled antibody conjugates for in vivo molecular imaging. Mol Imaging Biol 13(1):32-42

26. Xu H, Eck PK, Baidoo KE, Choyke PL, Brechbiel MW (2009) Toward preparation of antibody-based imaging probe libraries for dual-modality positron emission tomography and fluorescence imaging. Bioorg Med Chem 17(14):5176-5181

27. Paudyal P, Paudyal B, Iida Y, Oriuchi N, Hanaoka H, Tominaga H, Ishikita T, Yoshioka H, Higuchi T, Endo K (2009) Dual functional molecular imaging probe targeting CD20 with PET and optical imaging. Oncol Rep 22(1):115-119
28. Sampath L, Kwon S, Hall MA, Price RE, Sevick-Muraca EM (2010) Detection of cancer metastases with a dual-labeled near-infrared/positron emission tomography imaging agent. Transl Oncol 3(5):307-217

29. Baeuerle PA, Gires O (2007) EpCAM (CD326) finding its role in cancer. Br J Cancer 96(3):417-423

30. Epenetos AA, Canti G, Taylor-Papadimitriou J, Curling M, Bodmer WF (1982) Use of two epithelium-specific monoclonal antibodies for diagnosis of malignancy in serous effusions. Lancet 2(8306):1004-1006

31. Nagrath S, Sequist LV, Maheswaran S, Bell DW, Irimia D, Ulkus L, Smith MR, Kwak EL, Digumarthy S, Muzikansky A, Ryan P, Balis UJ, Tompkins RG, Haber DA, Toner M (2007) Isolation of rare circulating tumour cells in cancer patients by microchip technology. Nature 450 (7173):1235-1239

32. Ntouroupi TG, Ashraf SQ, McGregor SB, Turney BW, Seppo A, Kim Y, Wang X, Kilpatrick MW, Tsipouras P, Tafas T, Bodmer WF (2008) Detection of circulating tumour cells in peripheral blood with an automated scanning fluorescence microscope. Br J Cancer 99(5):789-795

33. Hall MA, Kwon S, Robinson H, Lachance PA, Azhdarinia A, Ranganathan R, Price RE, Chan W, Sevick-Muraca EM (2011) Imaging prostate cancer lymph node metastases with a multimodality contrast agent. Prostate. doi:10.1002/pros.21413

34. Zhou Y, Chakraborty S, Liu S (2011) Radiolabeled cyclic RGD peptides as radiotracers for imaging tumors and thrombosis by SPECT. Theranostics 1:58-82

35. Froidevaux S, Eberle AN, Christe M, Sumanovski L, Heppeler A, Schmitt JS, Eisenwiener K, Beglinger C, Macke HR (2002) Neuroendocrine tumor targeting: study of novel gallium-labeled somatostatin radiopeptides in a rat pancreatic tumor model. Int J Cancer 98(6):930-937

36. Kuhnast B, Bodenstein C, Haubner R, Wester HJ, SenekowitschSchmidtke R, Schwaiger M, Weber WA (2004) Targeting of gelatinase activity with a radiolabeled cyclic HWGF peptide. Nucl Med Biol 31 (3):337-344

37. Houston JP, Ke S, Wang W, Li C, Sevick-Muraca EM (2005) Quality analysis of in vivo near-infrared fluorescence and conventional gamma images acquired using a dual-labeled tumor-targeting probe. J Biomed Opt 10(5):054010

38. Ye Y, Bloch S, Xu B, Achilefu S (2008) Novel near-infrared fluorescent integrin-targeted DFO analogue. Bioconjug Chem 19(1):225-234

39. Edwards WB, Akers WJ, Ye Y, Cheney PP, Bloch S, Xu B, Laforest R, Achilefu S (2009) Multimodal imaging of integrin receptor-positive tumors by bioluminescence, fluorescence, gamma scintigraphy, and single-photon emission computed tomography using a cyclic RGD peptide labeled with a near-infrared fluorescent dye and a radionuclide. Mol Imaging 8(2):101-110

40. Bauer W, Briner U, Doepfner W, Haller R, Huguenin R, Marbach P, Petcher TJ, Pless (1982) SMS 201-995: a very potent and selective octapeptide analogue of somatostatin with prolonged action. Life Sci 31 (11):1133-1140

41. Krenning EP, Breeman WAP, Kooij PPM, Lameris JS, Bakker WH, Koper JW, Ausema L, Reubi JC, Lamberts SWJ (1989) Localisation of endocrine-related tumours with radioiodinated analogue of somatostatin. Lancet 333(8632):242-244

42. Bakker WH, Albert R, Bruns C, Breeman WA, Hofland LJ, Marbach P, Pless J, Pralet D, Stolz B, Koper JW et al (1991) [111In-DTPA-DPhe1]-octreotide, a potential radiopharmaceutical for imaging of somatostatin receptor-positive tumors: synthesis, radiolabeling and in vitro validation. Life Sci 49(22):1583-1591

43. Henze M, Schuhmacher J, Hipp P, Kowalski J, Becker DW, Doll J, Macke HR, Hofmann M, Debus J, Haberkorn U (2001) PET imaging of somatostatin receptors using [68GA]DOTA-D-Phe1-Tyr3-octreotide: first results in patients with meningiomas. J Nucl Med 42(7):1053-1056

44. Prasad V, Baum RP (2010) Biodistribution of the Ga-68 labeled somatostatin analogue DOTA-NOC in patients with neuroendocrine tumors: characterization of uptake in normal organs and tumor lesions. Q J Nucl Med Mol Imaging 54(1):61-67

45. Srirajaskanthan R, Kayani I, Quigley AM, Soh J, Caplin ME, Bomanji J (2010) The role of 68Ga-DOTATATE PET in patients with neuroendocrine tumors and negative or equivocal findings on 111In-DTPAoctreotide scintigraphy. J Nucl Med 51(6):875-882

46. Edwards WB, Xu B, Akers W, Cheney PP, Liang K, Rogers BE, Anderson CJ, Achilefu S (2008) Agonist-antagonist dilemma in molecular imaging: evaluation of a monomolecular multimodal imaging agent for the somatostatin receptor. Bioconjug Chem 19 (1): $192-200$ 
47. Ortega N, Behonick DJ, Werb Z (2004) Matrix remodeling during endochondral ossification. Trends Cell Biol 14(2):86-93

48. Rodenberg E, Azhdarinia A, Lazard Z, Hall M, Kwon S, Wilganowski N, Merched-Sauvage M, Salisbury EA, Davis AR, Sevick-Muraca EM, and Olmsted-Davis E (2011) MMP-9 as a biomarker of heterotopic ossification (in press)

49. Azhdarinia A, Wilganowski N, Robinson $\mathrm{H}$, Ghosh $\mathrm{P}$, Kwon $\mathrm{S}$, Lazard ZW, Davis AR, Olmsted-Davis E, Sevick-Muraca EM (2011) Characterization of chemical, radiochemical and optical properties of a dual-labeled MMP-9 targeting peptide. Bioorg Med Chem 19(12):3769-3776

50. Wang W, Ke S, Kwon S, Yallampalli S, Cameron AG, Adams KE, Mawad ME, Sevick-Muraca EM (2007) A new optical and nuclear dual-labeled imaging agent targeting interleukin 11 receptor alphachain. Bioconjug Chem 18(2):397-402

51. Lee H, Akers WJ, Cheney PP, Edwards WB, Liang K, Culver JP, Achilefu S (2009) Complementary optical and nuclear imaging of caspase-3 activity using combined activatable and radio-labeled multimodality molecular probe. J Biomed Opt 14(4):040507

52. Bhushan KR, Misra P, Liu F, Mathur S, Lenkinski RE, Frangioni JV (2008) Detection of breast cancer microcalcifications using a dualmodality SPECT/NIR fluorescent probe. J Am Chem Soc 130 (52): $17648-17649$

53. Meares CF, McCall MJ, Reardan DT, Goodwin DA, Diamanti CI, McTigue M (1984) Conjugation of antibodies with bifunctional chelating agents: isothiocyanate and bromoacetamide reagents, methods of analysis, and subsequent addition of metal ions. Anal Biochem 142 (1):68-78

54. Bjornsson OG, Murphy R, Chadwick VS (1982) Physiochemical studies of indocyanine green (ICG): absorbance/concentration relationship, $\mathrm{pH}$ tolerance and assay precision in various solvents. Experientia 38(12): 1441-1442

55. Buckle T, van Leeuwen AC, Chin PT, Janssen H, Muller SH, Jonkers J, van Leeuwen FW (2010) A self-assembled multimodal complex for combined pre- and intraoperative imaging of the sentinel lymph node. Nanotechnology 21(35):355101

56. van der Poel HG, Buckle T, Brouwer OR, Valdes Olmos RA, van Leeuwen FW (2011) Intraoperative laparoscopic fluorescence guidance to the sentinel lymph node in prostate cancer patients: clinical proof of concept of an integrated functional imaging approach using a multimodal tracer. Eur Urol 4:826-833

57. Williams ATR, Winfield SA, Miller JN (1983) Relative fluorescence quantum yields using a computer controlled luminescence spectrometer. Analyst 108:1067-1071

58. Sevick-Muraca EM, Heintzelman DL, Lee J, Troy TL, Paithankar DY (1997) Role of higher-order scattering in solutions to the forward and inverse optical-imaging problems in random media. Appl Opt 36 (34):9058-9067
59. Tarazi L, George A, Patonay G, Strekowski L (1998) Spectral characterization of a novel near-infrared cyanine dye: a study of its complexation with metal ions. Talanta 46(6):1413-1424

60. Decristoforo C, Knopp R, von Guggenberg E, Rupprich M, Dreger T, Hess A, Virgolini I, Haubner R (2007) A fully automated synthesis for the preparation of $68 \mathrm{Ga}$-labelled peptides. Nucl Med Commun 28 (11):870-875

61. Azhdarinia A, Wilganowski N, Robinson H, Ghosh P, Kwon S, Lazard Z, Davis AR, Olmsted-Davis E, and Sevick-Muraca EM (2011) Characterization of chemical, radiochemical and optical properties of a dual-labeled MMP-9 targeting peptide. Bioorg Med Chem (in press)

62. Li C, Wang W, Wu Q, Ke S, Houston J, Sevick-Muraca E, Dong L, Chow D, Charnsangavej C, Gelovani JG (2006) Dual optical and nuclear imaging in human melanoma xenografts using a single targeted imaging probe. Nucl Med Biol 33(3):349-358

63. Marshall MV, Draney D, Sevick-Muraca EM, Olive DM (2010) Singledose intravenous toxicity study of IRDye $800 \mathrm{CW}$ in Sprague-Dawley rats. Mol Imaging Biol 12(6):583-594

64. Rodenberg E, Azhdarinia A, Lazard ZW, Hall MA, Kwon SK, Wilganowski N, Salisbury E, Merched-Sauvage M, Davis EA, Sevick EM, Davis AR (2011) MMP-9 is a diagnostic marker of heterotopic ossification in a murine model. Tissue Eng Part A 17(19-20):2487-2496

65. Sampath L, Wang W, Sevick-Muraca EM (2008) Near infrared fluorescent optical imaging for nodal staging. J Biomed Opt 13 (4):041312

66. US Department of Health and Human Services, Food and Drug Administration, Center for Drug Evaluation and Research. Guidance, PET Drugs-Current Good Manufacturing Practice (CGMP) (2009) http://www.fda.gov/downloads/Drugs/GuidanceComplianceRegulatory Information/Guidances/ucm070306.pdf.

67. Zhang Z, Achilefu S (2005) Spectral properties of pro-multimodal imaging agents derived from a NIR dye and a metal chelator. Photochem Photobiol 81(6):1499-1504

68. Zhang Z, Liang K, Bloch S, Berezin M, Achilefu S (2005) Monomolecular multimodal fluorescence-radioisotope imaging agents. Bioconjug Chem 16(5):1232-1239

69. Garanger E, Aikawa E, Reynolds F, Weissleder R, Josephson L (2008) Simplified syntheses of complex multifunctional nanomaterials. Chem Commun (Camb) 39:4792-4794

70. Garanger E, Blois J, Hilderbrand SA, Shao F, Josephson L (2010) Divergent oriented synthesis for the design of reagents for protein conjugation. J Comb Chem 12(1):57-64

71. Ting R, Aguilera TA, Crisp JL, Hall DJ, Eckelman WC, Vera DR, Tsien RY (2010) Fast 18F labeling of a near-infrared fluorophore enables positron emission tomography and optical imaging of sentinel lymph nodes. Bioconjug Chem 21(10):1811-1819

72. Vera DR, Wisner ER, Stadalnik RC (1997) Sentinel node imaging via a nonparticulate receptor-binding radiotracer. J Nucl Med 38(4):530-535 